\title{
Analysis of Horn Antennas in Receiving Mode as an EM Field Sensor
}

\author{
Maifuz Ali ${ }^{1}$ and Seong-Ook Park ${ }^{2}$ \\ ${ }^{1}$ Information \& Electronics Research Institute, Department of Electrical Engineering, Korea Advanced Institute of Science \\ and Technology (KAIST), Daejon 305-701, Republic of Korea \\ ${ }^{2}$ Department of Electrical Engineering, Korea Advanced Institute of Science and Technology (KAIST), \\ Daejon 305-701, Republic of Korea
}

Correspondence should be addressed to Maifuz Ali, maifuzali@lycos.com

Received 20 July 2011; Revised 13 October 2011; Accepted 9 November 2011

Academic Editor: Krishnasamy Selvan

Copyright ( $) 2012$ M. Ali and S.-O. Park. This is an open access article distributed under the Creative Commons Attribution License, which permits unrestricted use, distribution, and reproduction in any medium, provided the original work is properly cited.

\begin{abstract}
Electromagnetic (EM) field measurement uses antenna in receiving mode as EM field sensor (EMFS). FDTD technique is applied to analyze the horn antenna in this mode. The performances of horn antenna as EMFS are predicted in terms of antenna factor (AF), and the results are compared with measurements. New empirical formula for quick calculation of antenna factor of pyramidal horn is also presented. Also, the gain of horn antennas are computed in receiving mode using FDTD technique, and the results are compared with published measured result and far-field gain formula.
\end{abstract}

\section{Introduction}

A key issue in the era of proliferating sensitive electronic instruments is the problem of electromagnetic interference (EMI). Accurate calibration of electromagnetic field sensor (EMFS) is required for EM field measurement. The frequency domain measurement performance of the sensor depends on its antenna factor (AF) which is the ratio of the incident electric field on the antenna surface to the received voltage across $50 \Omega$ load resistance $[1,2]$. The theoretical simulation of the AF of EMFS has attracted many research interests due to implementation cost and time required to calibrate a sensor experimentally [3-5]. Theoretical analysis of EMFS has been carried out till now using analytical technique and numerical techniques like method of moment (MoM). However, the FDTD technique which has found extensive use in electromagnetics $[6,7]$, to the best of the authors knowledge, has till now not been used explicitly in computing the AF of an EMFS. Also, the FDTD has the advantage that a single FDTD simulation can provide for both time domain and frequency domain analysis [6]. Further, the FDTD has the advantage that the problem of modeling a new structure is reduced to a problem of mesh generation rather than the complexity of reformulation of integral equations.

Commonly the measuring antennas or EMFS used are dipole and loop antenna. However, these are mostly effective up to a frequency of $1 \mathrm{GHz}[3]$. Beyond this range, a few waveguide-based sensors have been analyzed using MoM technique. In this work, waveguide-based horn antenna is analyzed in receiving mode for EMFS using standard FDTD technique and the obtained results are compared with measurement. New empirical formula for quick calculation of antenna factor of pyramidal horn is also presented.

In this case the knowledge of the effective area of the antenna is of great importance since the electromagnetic field power transmitted to the measuring unit is simply a product of the Pointing vector and the effective area. The effective area or antenna factor of the horn antenna is directly connected with the gain of the antenna. Also calculations of antenna gain is of interest to a wider group of the researchers. In this work, gain of horn antennas is computed in the receiving mode using the same FDTD technique and compared with the available gain formula in [8] and measured results of [9]. 


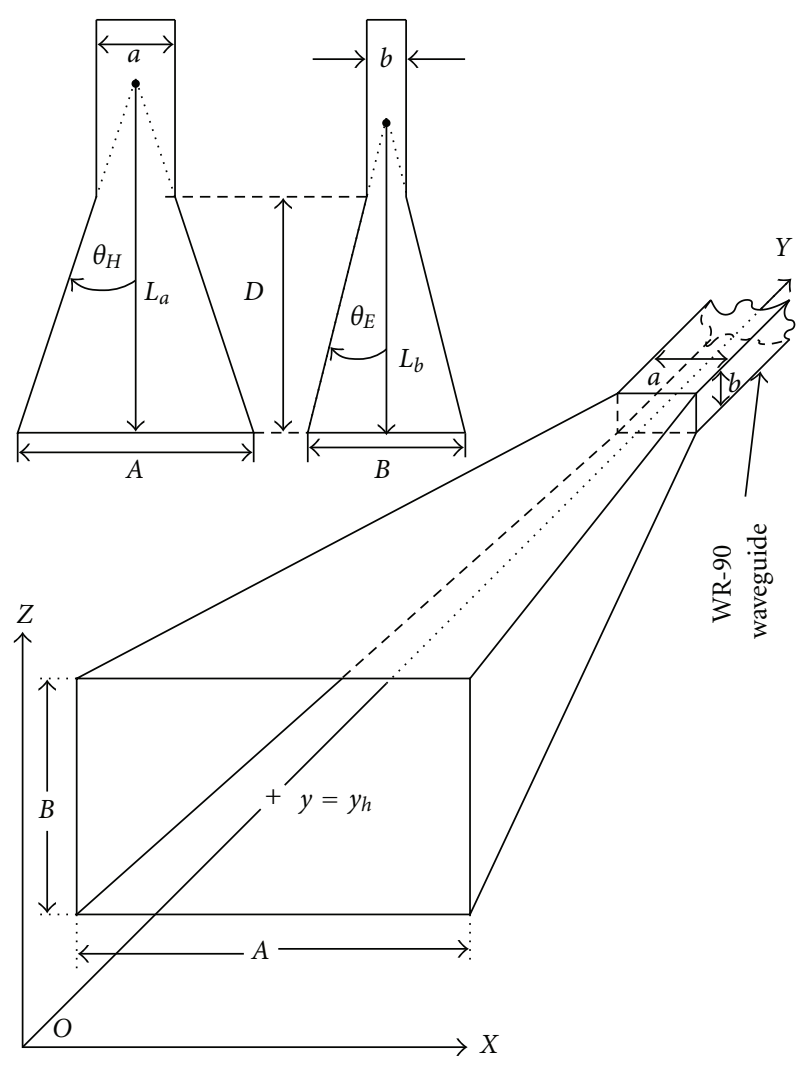

FIGURE 1: Details of geometry of a pyramidal horn antenna.

\section{FDTD Formulation of the Problem}

The FDTD model uses a uniform space lattice of cubic Yee cells having $\Delta x=\Delta y=\Delta z=\Delta$. A $10 \Delta$-thick unsplit perfectly matched layer (PML) $[10,11]$ is used as absorbing boundary conditions (ABC) on all six sides of the FDTD lattice. This PML is spaced $5 \Delta$ cells from the closest surface of the scatterer. Gaussian pulse $[7,10]$ is taken as the excitation source

$$
E_{z_{i, j, k}}(t)=A e^{-0.5\left(\left(t-t_{0}\right) / t_{\omega}\right)^{2}} .
$$

The Gaussian function maximizes to $A$ at $t=t_{0}$ and is zero at $t= \pm \infty$. $t_{\omega}$ is the standard deviation and relates the line width at half-height by the relationship

$$
t_{1 / 2}=\sqrt{8 \ln (2)} t_{\omega}=2.35482 t_{\omega}
$$

The complete geometry of the horn antenna is shown in Figure 1. The tangential electric field components along this structure are set to zero. Linearly polarized (along $z$-axis) perfectly plane wave of Gaussian pulse propagating along $y$-axis in free space incidents on the open end of the RW90 rectangular waveguide-fed horn at $\mathrm{y}=\mathrm{y}_{h}$ as shown in Figure 2.

In order to simulate a uniform plane wave within the FDTD lattice, the problem space is divided into the total field and scattered field regions and the antenna is placed within the total field regions. Details of this method given in [10] are used in this work. The plane wave is generated in the $X Z$-plane, at $y=y_{a}$. As primary and edge diffracted waves propagate through the horn waveguide structure, the subtractions in the $X Z$-plane at $y=y_{b}=y_{h}+1$ take place like in [10] but without the area $(A \times B)$ inside the horn at $y=y_{b}$.

As perfect plane wave and lossless free space are considered, time domain electric field at the aperture of the horn antenna is

$$
E_{z_{i, j, k}}(t)=A e^{-0.5\left(\left(t-t_{0}+t^{\prime}\right) / t_{\omega}\right)^{2}}
$$

where $t^{\prime}$ is the time shift due to the spacial difference between the antenna aperture and the position where Gaussian pulse is applied into the FDTD lattice. Fourier transform of $E_{z_{i, j, k}}^{t}$ gives the frequency domain incident electric field, which is

$$
E_{i}(\omega)=F\left\{E_{z_{i, j, k}}(t)\right\}
$$

where $F\{\}$ denotes the Fourier transform.

2.1. Power Flowing through the Waveguide. The time average complex power flowing through the waveguide is given by $[3,12]$

$$
P^{c}(\omega)=\int_{0}^{a} \int_{0}^{b} \frac{1}{2}\left[E(\omega) \times(H(\omega))^{*}\right] d x d z
$$

The integration is carried out over the waveguide cross section.

Although many modes are generated inside horn and enter into the waveguide after traveling few $\lambda$ distance through the waveguide, all the modes die down except $T E_{10}$ mode. Power calculating at the $X Z$-plane $\left(y=y_{0}\right)$ ensures that the measuring device receives only the dominant mode scattered power. Neglecting all components except $E_{z}$ and $H_{x}^{*}$, time average power flowing through the waveguide along $y$-direction is real part of $P^{c}(\omega)$ which is given by

$$
\begin{aligned}
P_{y}(\omega) & =\operatorname{Re}\left\{P^{c}(\omega)\right\} \\
& \approx \operatorname{Re}\left\{\left.\int_{0}^{a} \int_{0}^{b} \frac{1}{2} E_{z}(\omega) H_{x}(\omega)^{*}\right|_{y=y_{0}} d x d z\right\},
\end{aligned}
$$

where $y_{0}$ is the point in which all other modes are died down except $T E_{10}$ and a minimum 3 cell before the PML start within the FDTD space lattice. $a$ and $b$ are the dimensions of the feed waveguide shown in Figure 1.

2.2. Voltage Developed at the Matched Measuring Device. Since most measuring devices have an input impedance of $50 \Omega$, the voltage measured by these is given by

$$
V_{L}(\omega)=\sqrt{50 \times P_{y}(\omega)} \text { Volts }
$$

on the condition that the waveguide transporting this power is well matched with the measuring device. 


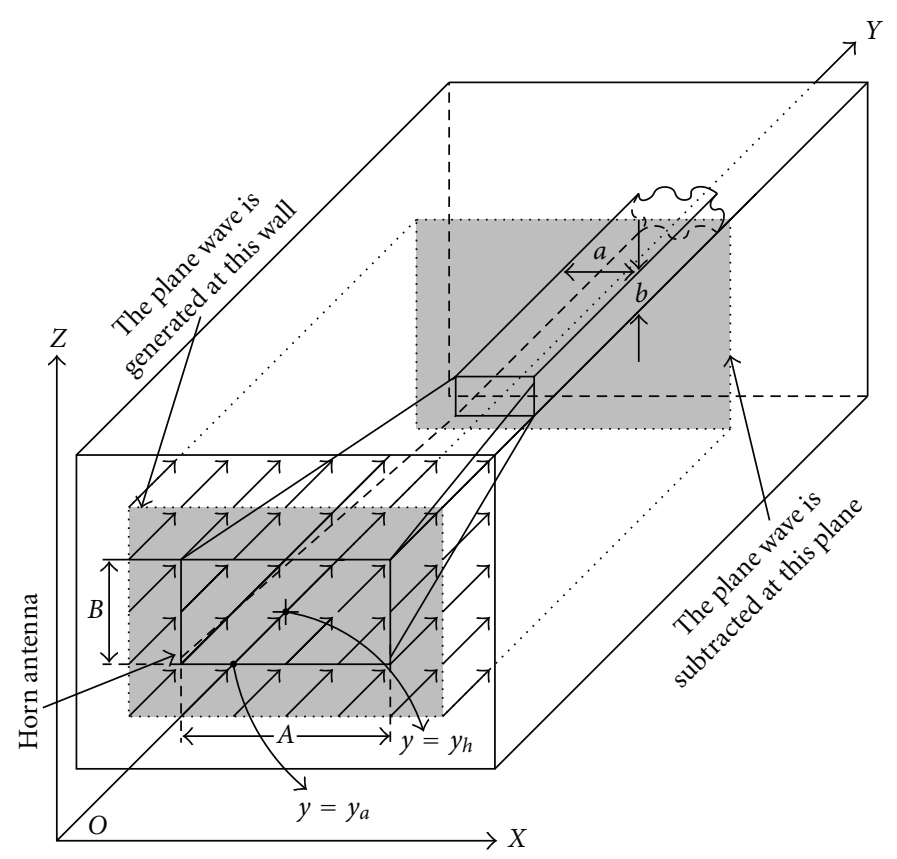

FIGURE 2: Receiving antenna case. A horn antenna under plane-wave illumination within the FDTD grid.

2.3. Calculation of Far-Field Antenna Factor. To carry out field strength measurements, one typically connects an antenna to a spectrum analyzer. The AF is the parameter that is used to convert the voltage or power reading of the receiver to the field strength incident on the antenna. In terms of an equation, the $\mathrm{AF}$ is defined as $[13,14]$

$$
\mathrm{AF}=\frac{E_{i}(\omega)}{V_{L}(\omega)} \mathrm{m}^{-1}=20 \log \left|\left(\frac{E_{i}(\omega)}{V_{L}(\omega)}\right)\right|\left[\mathrm{dB}\left(\mathrm{m}^{-1}\right)\right],
$$

where $E_{i}$ is the incident electric field on the surface of the antenna and $V_{L}$ is the voltage induced across a $50 \Omega$ load of the receiver.

During the progress of FDTD calculation time domain incident field $E_{z_{i, j, k}}(t)$ at $y=y_{h}$ and time domain electric field $E_{z}(t)$ and magnetic field $H_{x}(t)$ at $y=y_{0}$ on the surface area $a \times b$ are saved for each time step. The FDTD calculations are continued until all transients are dissipated, so that the Fourier transform yields the steady-state frequency domain response of the antenna. The frequency domain incident electric field is calculated from (4), and the voltage developed across $50 \Omega$ load $V_{L}(\omega)$ is obtained from (7). Finally, AF of the horn antenna is evaluated using (8). FDTD predicted AF flowing through this procedure takes the account of mutual coupling effects [15].

2.4. Calculation of Gain in Receiving Mode. A few widely used primary incident wave models for pyramidal horn antenna analysis are compared on the basis of gain calculations. The analysis based on edge-wave diffraction theory in [9] shows that these models agree well for high-gain horn with small flare angles but that the differences are more profound for lower gain horn, where the flare angles are larger. In this work, gain of various pyramidal horn antennas is calculated in receiving mode using FDTD technique.

Since the field strength can, alternatively, be measured in terms of antenna gain, the antenna factor can also be expressed as a function of antenna gain. If only the radiation far field is taken into account for antenna factor calculations, the antenna factor of the sensor is related to the gain by the formula $[15,16]$

$$
\mathrm{AF}=\frac{9.734}{\lambda \sqrt{G_{r}(\theta, \phi)}}
$$

where $G_{r}(\theta, \phi)$ is the antenna gain as a function of the angle of arrival of the energy and $\lambda$ is the free space wavelength in meter unit. From (8) and (9) gain of pyramidal horn is given by

$$
G=\frac{94.751}{\lambda^{2}}\left(\frac{V_{L}(\omega)}{E_{i}(\omega)}\right)^{2},
$$

where $E_{i}(\omega)$ and $V_{L}(\omega)$ are given in (4) and (7), respectively.

\section{Empirical Formula for Antenna Factor of Horn Antenna}

The gain of pyramidal horn antenna is calculated from $[8$, equation (18.4.2)], given by

$$
G=\frac{4 \pi}{\lambda^{2}}(A B) e\left(\sigma_{a}, \sigma_{b}\right) .
$$

$A$ and $B$ are the dimensions of the horn shown in Figure 1 and $e\left(\sigma_{a}, \sigma_{b}\right)$ is the aperture efficiency given in [8, equation (18.4.3)]. Other far-field gain formulas like Schelkunoff's 
horn-gain formula of [17] can also be used. But due to the availability of readily programs in [8] and accuracy, (11) is used for gain calculation in this work. From (9) and (11), AF of horn antenna is

$$
\mathrm{AF}=20 \log _{10}\left|\frac{9.734}{\sqrt{4 \pi A B e\left(\sigma_{a}, \sigma_{b}\right)}}\right|\left[\mathrm{dB}\left(\mathrm{m}^{-1}\right)\right] .
$$

\section{Numerical Results and Discussions}

Simulations are carried out in FDTD spatial grid with uniform cell size of $\Delta x=\Delta y=\Delta z=\Delta\left(\cong \lambda_{\min } / 24\right)=$ $1.0 \mathrm{~mm}$, where $\lambda_{\min }=24.0 \mathrm{~mm}$ is the wavelength at maximum frequency $12.4 \mathrm{GHz}$.

$\Delta t=1.67 p s$ is calculated from [10, equation (1.11)]. A linearly polarized (along $z$-axis) perfectly plane wave of Gaussian pulse having significant frequency content in a frequency range from $8 \mathrm{GHz}$ to $12.5 \mathrm{GHz}$ of maximum amplitude $A=1.0 \mathrm{~V} / \mathrm{m}, t_{0}=80.65 \mathrm{ps}$, and $t_{w}=10.08 \mathrm{ps}$ of (1) falls upon the open end of the horn at $y=y_{h}$.

The AF of two WR-90 waveguide-fed horns of different size are computed and compared with the experimental results. The experiment was carried out over the frequency range of $8 \mathrm{GHz}$ to $12.5 \mathrm{GHz}$ in step of $0.1 \mathrm{GHz}$. The experimental setup and procedure used in this work is given in detail in [3], and for this reason it is not repeated here.

\subsection{Antenna Factor of Pyramidal Horn Antennas}

4.1.1. Horn-1. The dimensions of the first horn analyzed as an EMFS are $A=14 \mathrm{~cm}, B=12 \mathrm{~cm}, D=18.5 \mathrm{~cm}, \theta_{H}=$ $17.57^{\circ}$ and $\theta_{E}=16.53^{\circ}$. FDTD computed AF is compared with the AF calculated from (12) and experimental result shown in Figure 3.

4.1.2. Horn-2. The dimensions of the second horn analyzed as an EMFS are $A=9 \mathrm{~cm}, B=7.9 \mathrm{~cm}, D=7.0 \mathrm{~cm}, \theta_{H}=$ $25.62^{\circ}$ and $\theta_{E}=26.17^{\circ}$. FDTD computed AF is compared with the AF calculated from (12) and experimental result shown in Figure 4.

From Figures 3 and 4, it is observed that the FDTD computed AF agrees well with the measured results. In Figure 4, AF computed using empirical formula (12) agrees on an average with the measurement and FDTD computed results. But in Figure 3, for comparatively high-gain horn, AF computed using empirical formula (12) has significant deviation from the measurement and FDTD computed results, mainly above $10.5 \mathrm{GHz}$. As the empirical formula assumes only far-field type fields, the deviation from this empirical formula underlines the effect of non-far-field type fields at the sensing plane of the sensor [3]. AF calculated from the empirical formula agrees with the measurement and FDTD calculation more for low-gain horn than for higher-gain horn.

4.2. Pyramidal Horn Antenna for Cross-Polarized Incident Field Detection. If the horn is rotated $\theta=90^{\circ}$ taking $y$-axis

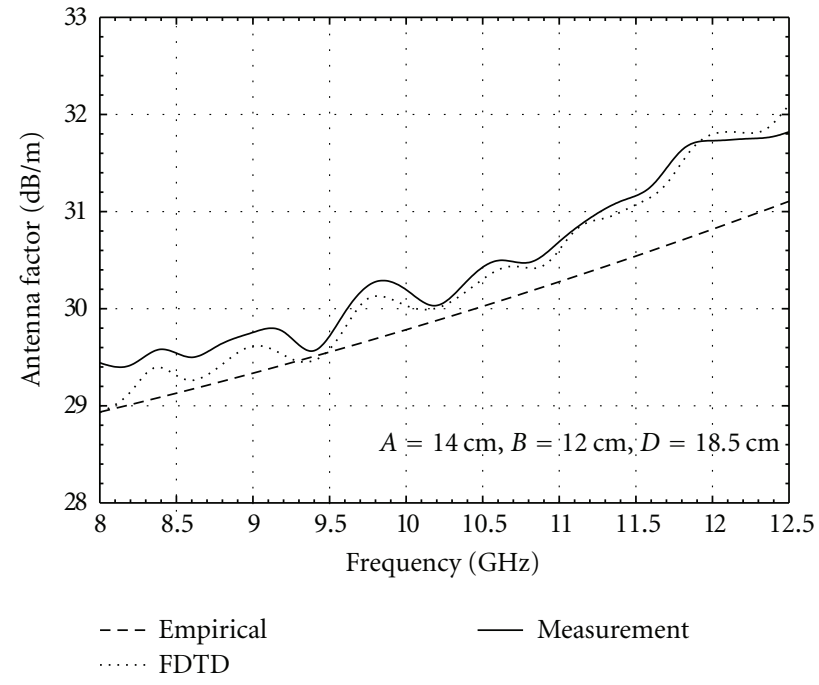

FIgURE 3: AF of pyramidal horn is computed using FDTD and empirical formula compared with the measurement.

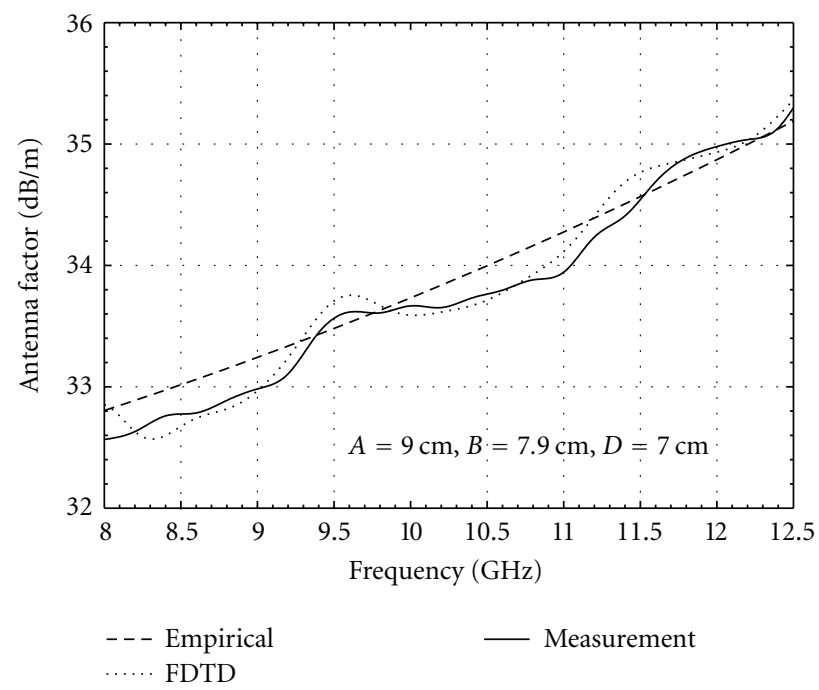

FIGURE 4: AF of pyramidal horn is computed using FDTD and empirical formula compared with the measurement.

as the axis of rotation, then the horn antenna becomes cross polarized with respect to the incident electric field. Then (6) for calculating time average power flowing through the waveguide along Y-direction becomes

$$
P(\omega) \approx \operatorname{Re}\left[\int_{0}^{a} \int_{0}^{b}-\left.\frac{1}{2} E_{x}(\omega)\left(H_{z}(\omega)\right)^{*}\right|_{y=y_{0}} d x d z\right]
$$

AF of the horn- 1 and horn- 2 of 4.1 are calculated for orthogonal incident electric field in the same way as in Section 4.1 and shown in Figure 5. AF of the horn in this position is greater than $100 \mathrm{~dB}$, though in ideal case $\mathrm{AF}=$ $\infty$. In practice, the received power is too low to separate itself from the EM noise present in the environment. For a good receiver the cross-polarization pick up of the antenna 


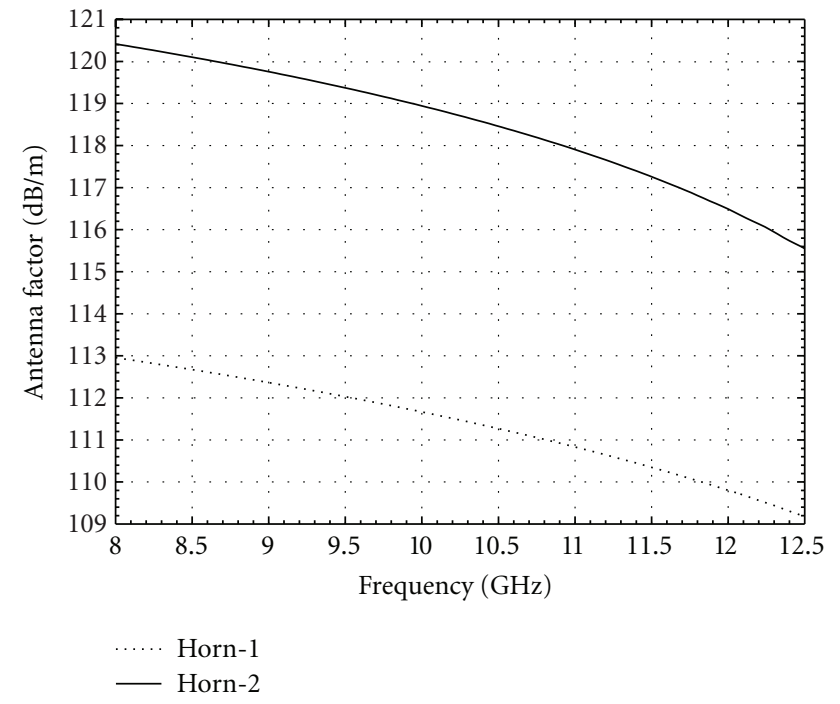

Figure 5: AF of pyramidal horn due to the incidence of crosspolarized electric field.

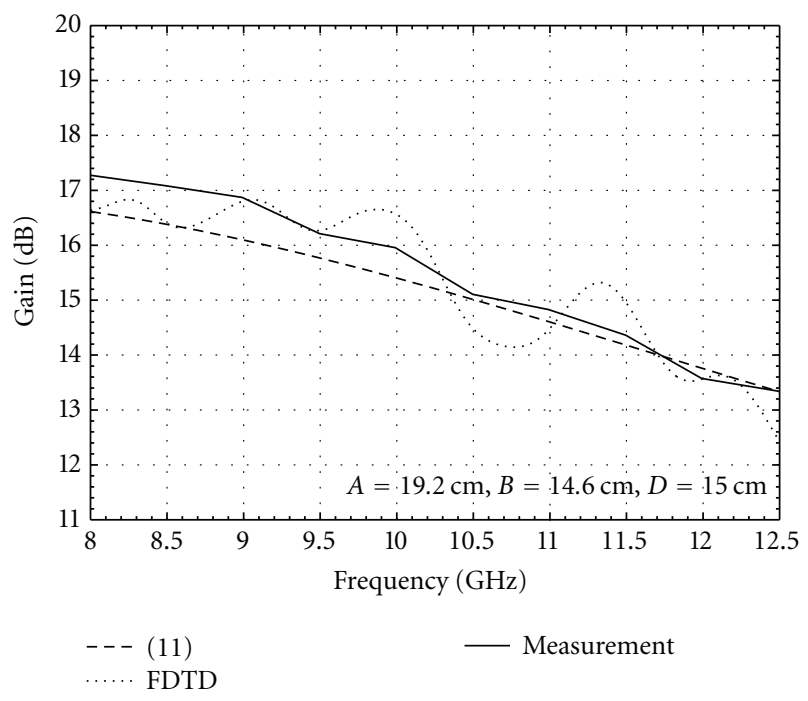

FIGURE 6: Gain of short horn computed using FDTD and compared with (11) and measurement of [9, Figure 2].

is expected to be minimum. Hence, this greater value of cross-polarization isolation, the performance of the antenna, is better as an EM field sensor [18].

4.3. Gain of Pyramidal Horn Antenna in Receiving Mode. The gain of two different pyramidal horn antennas are calculated using FDTD technique of (10) and compared with (11) and measured results of [9].

4.3.1. Horn-1. The dimensions of the first horn are $A=$ $19.2 \mathrm{~cm}, B=14.6 \mathrm{~cm}, D=15.0 \mathrm{~cm}, \theta_{H}=29.423^{\circ}$, and $\theta_{E}=$ $24.355^{\circ}$. The FDTD computed gain is compared with (11) and experimental results of [9, Figure 2] shown in Figure 6.

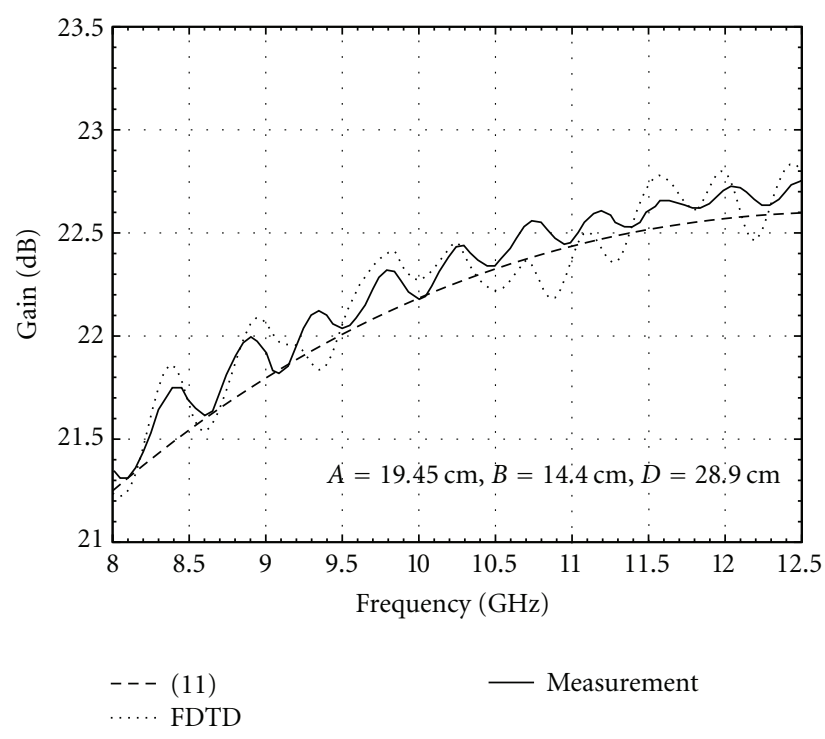

Figure 7: Gain of medium horn computed using FDTD and compared with (11) and measurement of [9, Figure 3].

4.3.2. Horn-2. The dimensions of the second horn are $A=$ $19.45 \mathrm{~cm}, B=14.4 \mathrm{~cm}, D=28.9 \mathrm{~cm}, \theta_{H}=16.539^{\circ}$, and $\theta_{E}=13.037^{\circ}$. The FDTD computed gain is compared with (11) and experimental results of [9, Figure 3] shown in Figure 7.

The results in Figure 6 are for horn with larger flare angles (short pyramidal horn), while the results in Figure 7 are for a medium pyramidal horn. From these results, it is seen that flare angles of the horns do not affect FDTD gain computation like in [9] and the results are well agreed with the measured values. Calculated gains using (11) match on an average with the FDTD computed results and measurements of [9], but no oscillation in magnitude is observed.

\section{Conclusions}

An empirical formula for AF of pyramidal horn antenna has been presented. AF obtained from measurements carried out for horn antennas of different sizes compares favorably with the FDTD predicted AF and AF calculated from the empirical formula (12). AF of the horn antenna is low which is desired for EM field sensor. The voltage developed at the receiver due to the cross-polarized component of incident field on the aperture of the pyramidal horn is found to be low and the resulting AF is high. Further the horn antenna has wide bandwidth and is therefore suitable as efficient EMFS.

The gain of pyramidal horns are computed in receiving mode using FDTD method, and results are compared with (11) and measured results of [9]. It can be concluded that the gain calculated using FDTD technique agrees well with measurement and not depends upon the horn shape like in [9].

Perfect coaxial match over the entire bandwidth is assumed for FDTD simulations. But in practice VSWR $\neq 1$ over the entire bandwidth. Due to this idealistic assumption, 
some difference is introduced between the FDTD simulated and measured results.

The amplitude oscillation in FDTD computed AF or gain due to the oscillation of amplitude in power flowing through the fed waveguide, calculated from (6), represents a standing wave component resulting from interference between the primary wave and the edge diffracted waves [19]. However, same variation is also observed in the measurements leading to the conclusion that it is an intrinsic antenna property. But this amplitude oscillation is not observed in the antenna gain using (11). As the empirical formula assumes only farfield type fields, the deviation from the empirical formula underlines the effect of non-far-field type fields.

\section{Acknowledgments}

The authors would like to thank Proffessor Subrata Sanyal, Proffessor A. Bhattacharya, and Proffessor Ajoy Chakraborty of the Indian Institute of Technology, Kharagpur, India, for critical discussion from time to time. This research was supported by the Korea Communications Commission (KCC) and National Research foundation (NRF) of Korea under Contract KCA-2011-1191304001-110010100 and 2011-0006570, respectively, and the Brain Korea 21 Project, the School of Information Technology, KAIST in 2011.

\section{References}

[1] H. Hosoyama, T. Iwasaki, and S. Ishigami, "Complex antenna factor of a V-dipole antenna with two coaxial feeders for field measurements," IEEE Transactions on Electromagnetic Compatibility, vol. 41, no. 2, pp. 154-158, 1999.

[2] S. Ishigami, H. Iida, and T. Iwasaki, "Measurements of complex antenna factor by the near-field 3-antenna method," IEEE Transactions on Electromagnetic Compatibility, vol. 38, no. 3, pp. 424-432, 1996.

[3] A. Bhattacharya, S. Gupta, and A. Chakraborty, "Analysis of rectangular waveguide and thick windows as EMI sensors," Progress in Electromagnetics Research, vol. 22, pp. 231-258, 1999.

[4] Y. Matsumoto, T. Umeda, A. Nishikata, K. Fujii, Y. Yamanaka, and A. Sugiura, "EMI antenna calibration on an absorberlined ground plane to determine free-space antenna factor," IEEE Transactions on Electromagnetic Compatibility, vol. 45, no. 4, pp. 656-660, 2003.

[5] K. Fujii, S. Harada, A. Sugiura, Y. Matsumoto, and Y. Yamanaka, "An estimation method for the free-space antenna factor of VHF EMI antennas," IEEE Transactions on Electromagnetic Compatibility, vol. 47, no. 3, pp. 627-634, 2005.

[6] A. Taflove and S. C. Hagness, Computational Electromagnetic-The Finite-Difference Time-Domain Method, Artech House, Boston, UK, 2005.

[7] W. Yu and R. Mittra, CFDTD: Conformal Finite-Difference Time-Domain Maxwell's Equations Solver: Software and User's Guide, Artech House, Boston, UK, 2004.

[8] S. J. Orfanidis, Electromagnetic Waves and Antennas, Rutgers University, 2008.

[9] G. Mayhew-Ridgers, J. W. Odendaal, and J. Joubert, "On primary incident wave models for pyramidal horn gain calculations," IEEE Transactions on Antennas and Propagation, vol. 48, no. 8, pp. 1246-1252, 2000.

[10] D. M. Sullivan, Electromagnetic Simulation Using The FDTD Method, IEEE Press, New York, NY, USA, 2000.

[11] D. M. Sullivan, "An unsplit step 3-D PML for use with the FDTD method," IEEE Microwave and Guided Wave Letters, vol. 7, no. 7, pp. 184-186, 1997.

[12] R. F. Harrington, Time-Harmonic Electromagnetic Fields, McGraw-Hill Book Company, New York, NY, USA, 1961.

[13] T. Iwasaki and K. Tomizawa, "Systematic uncertainties of the complex antenna factor of a dipole antenna as determined by two methods," IEEE Transactions on Electromagnetic Compatibility, vol. 46, no. 2, pp. 234-245, 2004.

[14] W. Joseph and L. Martens, "An improved method to determine the antenna factor," IEEE Transactions on Instrumentation and Measurement, vol. 54, no. 1, pp. 252-257, 2005.

[15] R. M. Hekert, J. K. Daher, K. P. Ray, and B. Subbarao, "Measurementand modeling of near and far field antenna factor," in Proceedings of the International Conference on Electromagnetic Compatibility and Interference (INCEMIC '94), pp. 237-241, August 1994.

[16] W. S. Cho, M. Kanda, H. J. Hwang, and M. W. Howard, "A disk-loaded thick cylindrical dipole antenna for valuation of an EMC test site from 30 to $300 \mathrm{MHz}$," IEEE Transactions on Electromagnetic Compatibility, vol. 42, no. 2, pp. 172-180, 2000.

[17] K. T. Selvan, "An approximate generalization of schelkunoff s horn-gain formulas," IEEE Transactions on Antennas and Propagation, vol. 47, no. 6, pp. 1001-1004, 1999.

[18] S. Ghosh and A. Chakrabarty, "Ultrawideband performance of dielectric loaded T-shaped monopole transmit and receive antenna/EMI sensor," IEEE Antennas and Wireless Propagation Letters, vol. 7, Article ID 921335, pp. 358-361, 2008.

[19] J. F. Nye and W. Liang, "Theory and measurement of the field of a pyramidal horn," IEEE Transactions on Antennas and Propagation, vol. 44, no. 11, pp. 1488-1498, 1996. 

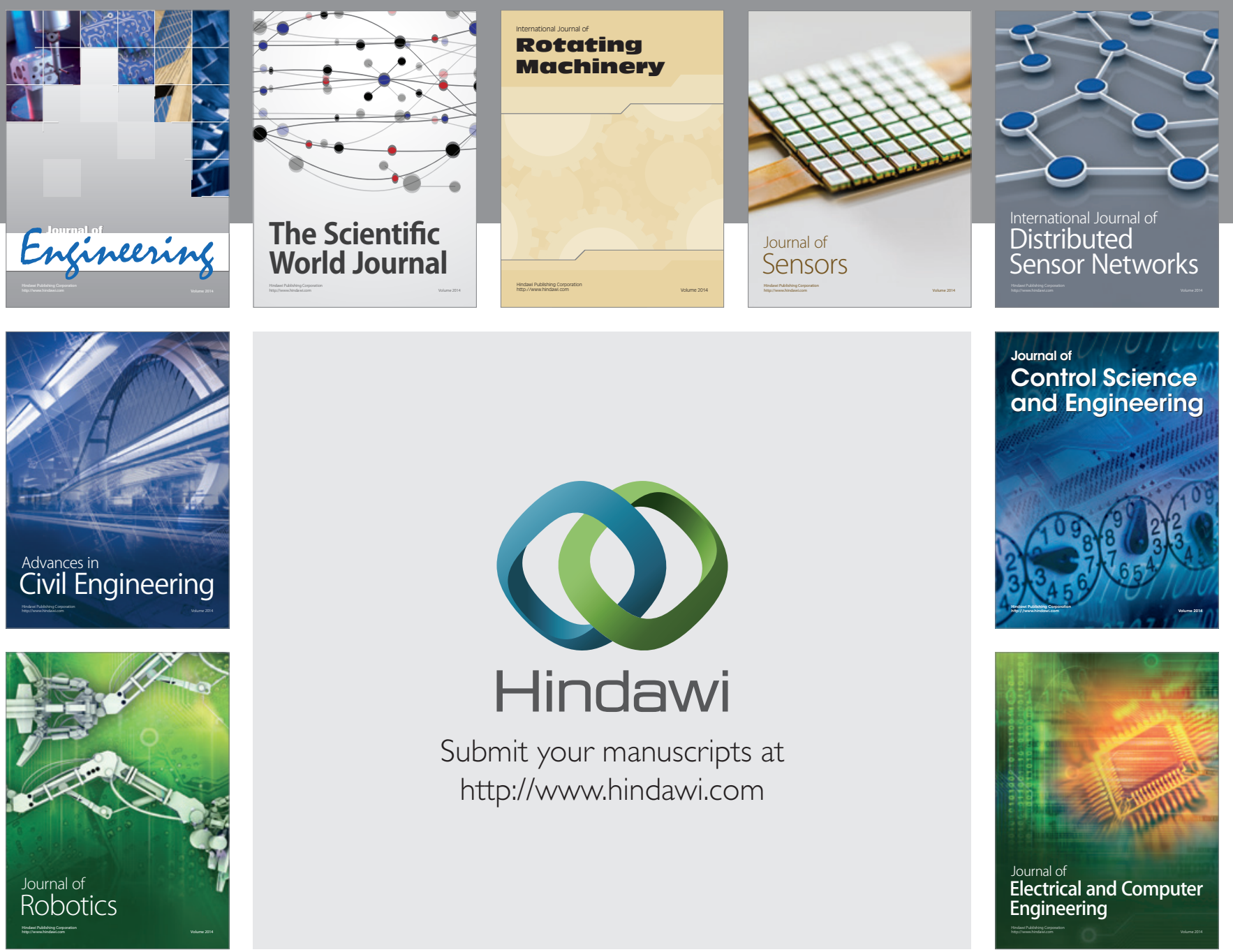

Submit your manuscripts at

http://www.hindawi.com
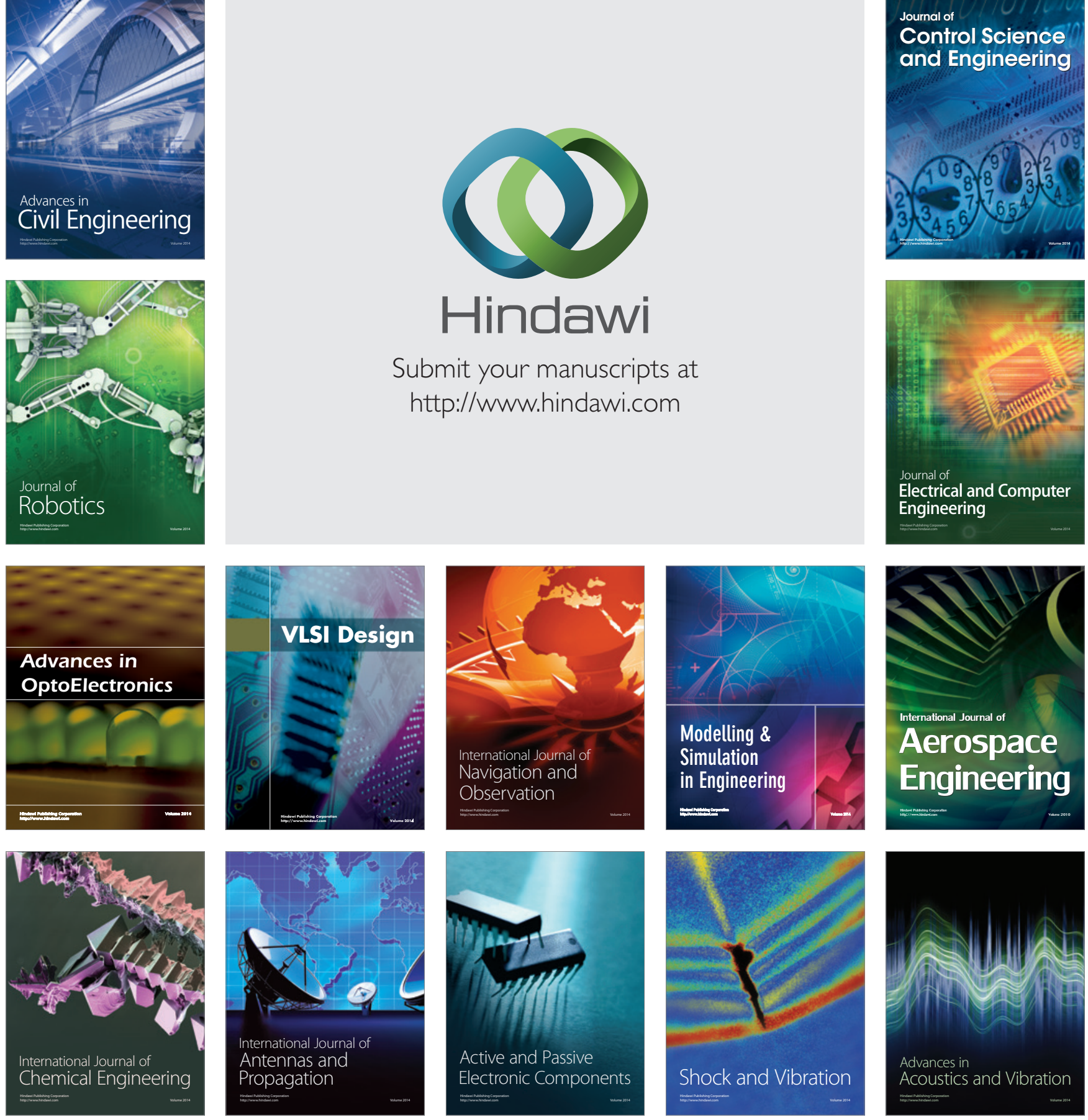